\title{
Time-periodic Poiseuille flow in a pipe for some classes of fluids
}

\author{
Alessandra Borrelli · Maria Cristina Patria
}

Published online: 6 November 2007

(C) Università degli Studi di Ferrara 2007

\section{Erratum to: Ann Univ Ferrara (2007) 53(1):13-28 DOI 10.1007/s11565-007-0002-4}

The original version of this article unfortunately contained a mistake.

In page 19, lines 18-19, the sentence "Then the problem (2.1), (3.1), (3.2)" should be changed to "Then the problem (2.1) with $\boldsymbol{\Gamma}(t)=\Gamma(t) \mathbf{h}$ and $\Phi(t)=\int_{\Sigma} \mathbf{h} \cdot \mathbf{V}(\mathbf{x}, t) d \Sigma$ "

In page 20 , line 7 , equation "V $\equiv \mathbf{V}(\mathbf{x})=\frac{\Phi}{a_{01}} \varphi_{0}(\mathbf{x})$ " should be changed to $" \mathbf{V} \equiv \mathbf{V}(\mathbf{x})=\frac{\Phi}{\mathbf{h} \cdot \mathbf{a}_{0}} \varphi_{0}(\mathbf{x}) "$.

The online version of the original article can be found under doi:10.1007/s11565-007-0002-4.

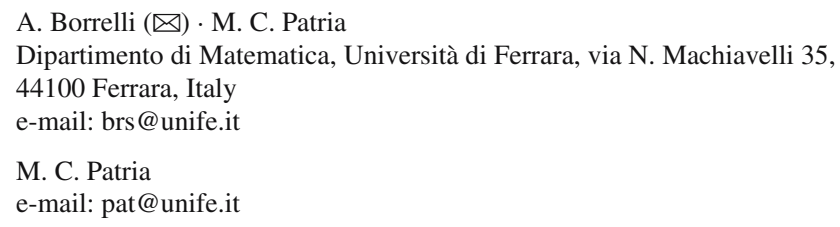

